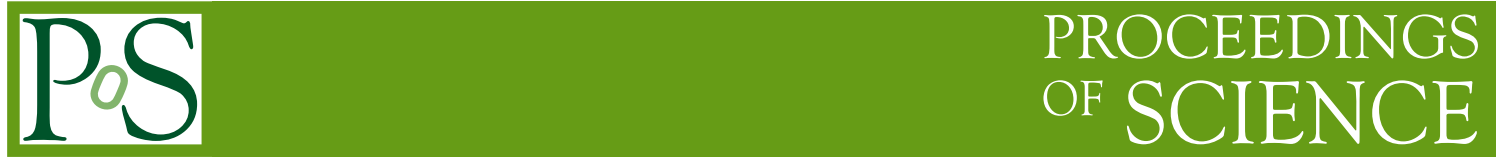

\title{
Diseases with rooted staggered quarks
}

\author{
Michael Creutz* \\ Brookhaven National Laboratory, Upton, NY 11973, USA \\ E-mail: creutz@bnl.gov
}

\begin{abstract}
Calculations using staggered quarks augmented with a root of the fermion determinant to reduce doubling give a qualitatively incorrect behavior in the small quark mass region. Attempts to circumvent this problem for the continuum limit involve an unproven combination of unphysical states, a loss of unitarity, and a rather peculiar non-commutation of limits.
\end{abstract}

XXIV International Symposium on Lattice Field Theory

July 23-28 2006

Tucson Arizona, US

\footnotetext{
${ }^{*}$ Speaker.
} 


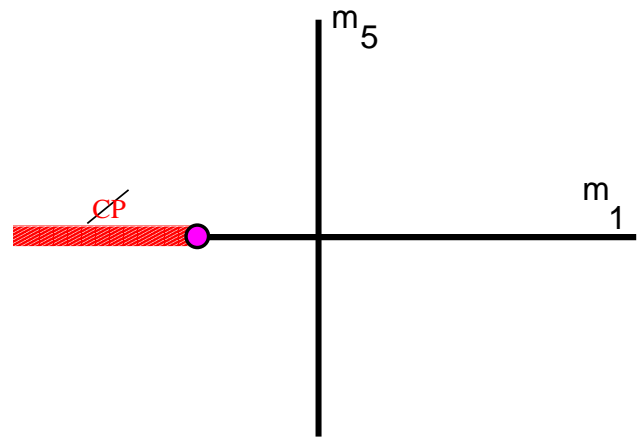

Figure 1: The phase diagram for one flavor quark-gluon dynamics as a function of $m_{1}$ and $m_{5}$ defined in the text. A first order phase transition line ending at a second order phase transition appears along the negative mass axis. The transition represents a spontaneous breaking of CP symmetry. A finite gap separates the transition from vanishing quark mass.

A popular approximation $[1,2]$ in lattice gauge theory arises from the simplicity of the staggered fermion formulation $[3,4,5]$. With only one Dirac component on each site, the large matrix inversions involved with conventional algorithms are substantially faster than with other approaches. However the approach and its generalizations are based on a discretization method that inherently requires a multiple of four fundamental fermions. The reasons for this are related to the cancellation of chiral anomalies. To apply the technique to the physical situation of two light and one intermediate mass quark requires an extrapolation down in the number of fermions.

As usually implemented, the approach involves taking a root of the fermion determinant inside standard hybrid Monte Carlo simulation algorithms. This step has not been justified theoretically. Here I argue that at finite lattice spacing this gives a qualitatively incorrect behavior as a function of the quark mass. To produce a correct continuum limit requires the removal of unphysical singularities which persist at finite volume and in regions of parameter space where there are no physical massless particles. Even in regions where the fermion determinant is positive definite, the procedure imposes non-trivial Ward identities incorporating unphysical states.

The basic problem arises from the exact chiral symmetry of staggered quarks. This symmetry appears for each flavor independently and survives the rooting procedure. Due to well known anomalies, this symmetry can exceed that allowed in the target theory.

The issues are sharpest in one flavor QCD. This theory has a rather interesting phase diagram when considered as a function of the quark mass $[6,7,8]$. In particular, consider the generalized mass term in continuum notation

$$
m_{1} \bar{\psi} \psi+i m_{5} \bar{\psi} \gamma_{5} \psi
$$

As a function of $m_{1}$ and $m_{5}$, this model has a first order phase transition along a portion of the negative $m_{1}$ axis, as sketched in Fig.(1). In many discussions $m_{1}$ and $m_{5}$ are combined to define a "complex" mass $m=m_{1}+i m_{5}$.

The $m_{5}$ term introduces an explicit breaking of $C P$ symmetry. When $m_{5}$ vanishes, the first order transition along the negative mass axis represents a spontaneous breakdown of this symmetry. A convenient order parameter is the pseudoscalar expectation

$$
\left\langle\eta^{\prime}\right\rangle=i\left\langle\bar{\psi} \gamma_{5} \psi\right\rangle
$$


This quantity displays a finite jump in sign as one passes through the transition.

This phase diagram illustrates several important features of one flavor QCD. First, the naive classical symmetry under $m \rightarrow-m$ is lost, having been broken by the anomaly. Second, this transition line does not start at $m=0$ but is separated from that point by a finite gap. Indeed, physics is expected to be analytic in the mass for a finite region around the origin. The main point I am raising is that these features are missing with rooted staggered quarks at finite lattice spacing. For the correct physics to be recovered in the continuum would require a complex and implausible cancellation of unphysical effects.

I briefly review the rooted staggered quark approach. Starting with naive lattice fermions, well known doubling issues give the fermion spectrum a sixteen fold degeneracy. The staggered approach reduces this degeneracy using the observation that for any closed fermion loop, the product of gamma matrices associated with that loop is proportional to the identity. If a fermion loop starts with only one non-vanishing component on any site, it always involves only a single component on each site it passes through. The sixteen doublers immediately factor into four independent pieces. This can be formalized by introducing a projection operator on each site

$$
\begin{array}{r}
\psi \rightarrow P \psi \\
P=P^{2} \\
\operatorname{Tr} P=1
\end{array}
$$

A particular implementation of this is

$$
P=\frac{1}{4}\left(1+i \gamma_{1} \gamma_{2}(-1)^{x_{1}+x_{2}}+i \gamma_{3} \gamma_{4}(-1)^{x_{3}+x_{4}}+\gamma_{5}(-1)^{x_{1}+x_{2}+x_{3}+x_{4}}\right)
$$

where the $x_{i}$ are the integer coordinates of the lattice site in question. This projection reduces the degeneracy from sixteen to four.

To reduce the doubling further requires something additional, and this is where the rooting "trick" comes in. Formally the procedure starts with the path integral and replaces the fermion determinant with its fourth root, i.e. $|D| \rightarrow|D|^{1 / 4}$. In practice this is achieved by inserting a factor of $1 / 4$ in the fermion force term while traversing a hybrid Monte Carlo trajectory. As $D$ still only involves a single fermion component on each site, the process remains quite frugal with computer time. Indeed, this is the main motivation for the scheme.

The problems with this stem from an exact symmetry of the unrooted theory that should not be there for the one flavor model. To see this symmetry explicitly in terms of the basic parameters, generalize the staggered mass term to

$$
\left(m_{1}+i S(x) m_{2}\right) \bar{\psi}(x) \psi(x)
$$

where $S(x)$ represents the parity of the site in question,

$$
S(x)=(-1)^{x_{1}+x_{2}+x_{3}+x_{4}} .
$$

With this extension, the fermion determinant and therefore the path integral are exactly invariant under the transformation

$$
\left(\begin{array}{l}
m_{1} \\
m_{2}
\end{array}\right) \longrightarrow\left(\begin{array}{cc}
\cos (\theta) & \sin (\theta) \\
-\sin (\theta) & \cos (\theta)
\end{array}\right)\left(\begin{array}{l}
m_{1} \\
m_{2}
\end{array}\right)
$$


As the mass becomes small, the symmetry requires the appearance of a Goldstone boson or parity doubling. For the unrooted theory this is not a problem. The various doublers rotate differently under chiral symmetry, and this represents a non-singlet axial symmetry. The Goldstone boson is one of the expected pseudoscalar bound states of the four fermion "tastes."

Is there some connection between the parameter $m_{5}$ in Eq. (1) and the parameter $m_{2}$ in Eq. (7)? Naively there would seem to be since the projection operator satisfies

$$
\gamma_{5} P=S(x) P
$$

However for the unrooted theory the rotation in Eq. (9) is a flavored axial symmetry; so, there is a hidden flavor matrix in Eq. (7). For the rooted theory this symmetry is spurious, the theories are different, and the question of this paragraph is moot.

To make the problem with rooting more precise, consider working in finite volume and on a lattice with an even number of sites. In that case the determinant of the fermion matrix $|D|$ is a polynomial in the combination $m_{1}^{2}+m_{2}^{2}$. This is easily seen from the hopping parameter expansion. Furthermore $|D|$ is real and non-vanishing for real $m_{1}^{2}+m_{2}^{2}>0$. This comes by looking at the eigenvalues of $D$ after rotating away $m_{2}$ in favor of $m_{1}$. Finally, there is a lower bound on the determinant

$$
|D| \geq\left(m_{1}^{2}+m_{2}^{2}\right)^{N_{c} V / 2}
$$

where $N_{c}$ is the number of colors and $V$ the number of lattice sites.

These facts show that roots of the determinant are analytic in $m_{1}$ and $m_{2}$ for at least some finite vicinity of any point where $m_{1}^{2}+m_{2}^{2}>0$. This analyticity allows a continuation from $m$ to $-m$. For example one can follow the path

$$
m_{1}=m \cos (\theta), \quad m_{2}=m \sin (\theta), \quad 0 \leq \theta \leq \pi .
$$

This continuation avoids all singularities in $|D|^{1 / 4}$.

We conclude that for rooted staggered fermions all correlations at $m_{1}$ are identical to those at $-m_{1}$. Furthermore the Ward identities that follow from $\frac{d^{n}}{d^{n} \theta} Z=0$ require a Goldstone boson or parity doubling. These properties are in direct contradiction to the known behavior of the one flavor theory, which is analytic at $m=0$ with the $m$ and $-m$ theories being inequivalent and there is no Goldstone boson or parity doubling. While the one flavor theory is somewhat peculiar, these problems extend directly to more flavors with non-degenerate quarks. This is because one has an independent $U(1)$ symmetry for each flavor, which is one more symmetry than allowed.

The advocates for staggered quarks $[9,10,11]$ have argued that these facts would be irrelevant if the diseases were to go away in the limit of vanishing lattice spacing. They argue that the the limits $a \rightarrow 0$ and $m \rightarrow 0$ might not commute. They point out that instanton effects can bring in $\sqrt{m^{2}+a^{2}}$ factors which break the $m \rightarrow-m$ symmetry if $a \rightarrow 0$ first. They do allow that there are extra Ward identities, but these could be satisfied by indefinite metric "taste" states. The required Goldstone boson would be there, but as one of a larger set of unphysical states. The use of an indefinite metric could permit a cancellation of these extra states from all physical processes.

Is this picture for saving the rooting procedure plausible? The idea of infrared and ultraviolet limits not commuting seems a bit strange, but the staggered proponents are asking a lot more. The problems persist even at finite volume, where one would not expect any infrared issues with the 
mass going to zero. Furthermore, for the one flavor theory the physical spectrum does not contain any massless particles; so, it is unclear why an infrared regulator should be required anyway.

Note that the chiral behavior at finite cutoff $a$ is explicitly wrong because of the extra Goldstone boson; indeed, this invalidates what was once regarded as a virtue of staggered fermions, the fact that they have some remnant chiral symmetry. Furthermore, the use of an indefinite metric for unphysical states requires a major leap from the canonical lattice ideas of Osterwalder and Seiler [12]. Finally one should note that these issues are not present with other fermion regulators, such as Wilson [13], domain wall [14], or the overlap [15].

In conclusion, at finite lattice spacing rooted staggered quarks give a qualitatively incorrect description of one flavor QCD. Attempts to circumvent this require complicated and implausible limits, not necessary with other lattice regulators. Without proof, rooted staggered quarks should be regarded as an uncontrolled approximation, from which claims of first principles calculations are at best premature.

\section{Acknowledgments}

This manuscript has been authored under contract number DE-AC02-98CH10886 with the U.S. Department of Energy. Accordingly, the U.S. Government retains a non-exclusive, royaltyfree license to publish or reproduce the published form of this contribution, or allow others to do so, for U.S. Government purposes.

\section{References}

[1] C. Aubin et al. [Fermilab Lattice Collaboration], Phys. Rev. Lett. 94, 011601 (2005) [arXiv:hep-ph/0408306].

[2] C. Aubin et al. [MILC Collaboration], Phys. Rev. D 70, 114501 (2004) [arXiv:hep-lat/0407028].

[3] J. B. Kogut and L. Susskind, Phys. Rev. D 11, 395 (1975).

[4] L. Susskind, Phys. Rev. D 16, 3031 (1977).

[5] H. S. Sharatchandra, H. J. Thun and P. Weisz, Nucl. Phys. B 192, 205 (1981).

[6] P. Di Vecchia and G. Veneziano, Nucl. Phys. B 171 (1980) 253.

[7] H. Leutwyler and A. Smilga, Phys. Rev. D 46, 5607 (1992).

[8] M. Creutz, Rev. Mod. Phys. 73, 119 (2001) [arXiv:hep-lat/0007032].

[9] C. Bernard, M. Golterman, Y. Shamir and S. R. Sharpe, arXiv:hep-lat/0603027.

[10] S. Durr and C. Hoelbling, Phys. Rev. D 71, 054501 (2005) [arXiv:hep-lat/0411022].

[11] S. Durr and C. Hoelbling, Phys. Rev. D 74, 014513 (2006) [arXiv:hep-lat/0604005].

[12] K. Osterwalder and E. Seiler, Annals Phys. 110, 440 (1978).

[13] K. G. Wilson, New Phenomena In Subnuclear Physics. Part A. Proceedings of the First Half of the 1975 International School of Subnuclear Physics, Erice, Sicily, July 11 - August 1, 1975, ed.

A. Zichichi, Plenum Press, New York, 1977, p. 69, CLNS-321

[14] V. Furman and Y. Shamir, Nucl. Phys. B 439, 54 (1995) [arXiv:hep-lat/9405004].

[15] H. Neuberger, Phys. Lett. B 417, 141 (1998) [arXiv:hep-lat/9707022]. 